\title{
Domain-general Stroop Performance and Hemispheric Asymmetries: A Resting-state EEG Study
}

\author{
Ettore Ambrosini ${ }^{1}$ and Antonino Vallesi ${ }^{1,2}$
}

\begin{abstract}
The ability to suppress irrelevant information while executing a task, also known as interference resistance ability, is a function of $\mathrm{pFC}$ that is critical for successful goal-directed human behavior. In the study of interference resistance and, more generally, executive functions, two key questions are still open: Does pFC contribute to cognitive control abilities through lateralized but domain-general mechanisms or through hemispheric specialization of domain-specific processes? And what are the underlying causes of interindividual differences in executive control performance? To shed light on these issues, here we employed an interindividual difference approach to investigate whether participants' hemispheric asymmetry in resting-state electrophysiological brain dynamics may reflect their variability in domain-general interference resistance. We recorded participants' resting-state electroencephalographic activity and performed spectral power analyses on the estimated cortical source activity.
\end{abstract}

\section{INTRODUCTION}

Executive functions are the set of cognitive processes that enable us to achieve internally represented goals by flexibly regulating thoughts and behaviors (Koechlin, Ody, \& Kouneiher, 2003; Miller \& Cohen, 2001; Stuss, Shallice, Alexander, \& Picton, 1995). This ability of cognitive control is particularly required in new or cognitively demanding situations, or when prepotent response tendencies must be overcome (MacDonald, Cohen, Stenger, \& Carter, 2000; Burgess \& Shallice, 1996). An essential component of cognitive control indeed involves the ability to boost the processing of task-relevant information while suppressing that of dominantly represented but irrelevant and even potentially distracting information (i.e., interference resistance). This process (and, in general, cognitive control) is commonly investigated using the Stroop paradigm (MacLeod, 1991; Stroop, 1935). The critical measure is the so-called Stroop effect, which refers to the robust performance decline in reporting task-relevant, but relatively weakly represented, features of stimuli that also contain task-irrelevant but ${ }^{1}$ University of Padua, ${ }^{2}$ IRCCS San Camillo Hospital Foundation,
Lido-Venice, Italy
To measure participants' lateralized brain dynamics at rest, we computed the right-left hemispheric asymmetry score for the $\beta / \alpha$ power ratio. To measure their domain-general interference resistance ability, verbal and spatial Stroop tasks were used. Robust correlations followed by intersection analyses showed that participants with stronger resting-state-related left-lateralized activity in different $\mathrm{pFC}$ regions, namely the mid-posterior superior frontal gyrus, middle and posterior middle frontal gyrus, and inferior frontal junction, were more able to inhibit irrelevant information in both domains. The present results confirm and extend previous findings showing that neurophysiological difference factors may explain interindividual differences in executive functioning. They also provide support for the hypothesis of a left pFC hemispheric specialization for domainindependent phasic cognitive control processes mediating Stroop performance. prepotent features as compared with those that do not. How our brain mediates executive functioning is one of the most fascinating and challenging questions facing cognitive neuroscience, and the past 30 years have witnessed a considerable improvement of our understanding of the processes enabling cognitive control (e.g., Miller \& Cohen, 2001; Duncan \& Owen, 2000; Norman \& Shallice, 1986). However, as noted by Braver (2012), "the majority of research efforts have focused on accounting for the diversity, scope and range of cognitive control functions in terms of an ever expanding conceptual taxonomy or fine-grained anatomically oriented fractionation scheme," and we might "not see the forest through the trees" (Cabeza \& Nyberg, 2003). Indeed, there is still a great deal that remains inadequately understood or debated among researchers. In particular, despite the increasing use of interindividual difference approaches in cognitive neuroscience studies of executive functions (Braver, Cole, \& Yarkoni, 2010), the origins of the huge individual differences in executive control performance remain poorly understood. In a recent work aiming to unveil what determines individual differences in executive functioning (Ambrosini \& Vallesi, 2016), we showed that the interindividual variability in task-switching ability, which is mediated by phasic cognitive control 
processes, is related to the degree of prefrontal hemispheric asymmetry in intrinsic (i.e., resting-state-related) brain dynamics, as assessed by the participants' sourcebased EEG spectral profile at rest. We suggested that a more strongly left-lateralized prefrontal intrinsic activity may represent a stable individual difference factor leading to biases in engaging the left-lateralized, phasic cognitive control processes to regulate goal-directed behavior.

Here, by using the same interindividual difference approach, we sought to verify whether the same specific neurophysiological individual difference factor (i.e., the prefrontal hemispheric asymmetry in EEG spectral profile at rest) may also explain the interindividual variability in interference resistance. Our hypothesis was based on the general underlying idea that executive functions may be dissociable anatomically, along the left-right axis of $\mathrm{pFC}$ and related neural networks, as proposed by the ROBBIA (ROtman-Baycrest Battery to Investigate Attention) model of executive functions. This model was based on vast neuropsychological (Stuss, 2011; Shallice, Stuss, Picton, Alexander, \& Gillingham, 2007, 2008; Stuss \& Alexander, 2007) and neuroimaging (see Vallesi, 2012, for a review) evidence. In particular, this model proposes a prefrontal hemispheric specialization of two distinct executive functions: the right-lateralized monitoring and the leftlateralized criterion setting (or task setting). The latter can be defined as the phasic, transient cognitive control processes needed to set up or select task-relevant rules and information (Stuss \& Alexander, 2007) and suppress the task-irrelevant ones that may interfere with accomplishing the goal-driven task at hand (Vallesi, McIntosh, Crescentini, \& Stuss, 2012; Fletcher, Shallice, \& Dolan, 2000; Thompson-Schill, D'Esposito, Aguirre, \& Farah, 1997); as such, criterion-setting processes would mediate both the task-switching and interference resistance abilities. Therefore, we expected the participants' Stroop performance to be mediated by the same neurophysiological individual difference factor (i.e., the prefrontal hemispheric asymmetry in EEG spectral profile at rest) that we showed to mediate their task-switching performance (Ambrosini \& Vallesi, 2016). However, to safely argue for the hypothesized (left) prefrontal hemispheric asymmetry of criterionsetting processes mediating Stroop performance, it must be verified that it does not simply depend on verbal processing, which is known to be left-lateralized (Hellige, 1993). Nonetheless, most of the previous studies on interference resistance used the classic color-word Stroop task, which strongly relies on left-lateralized verbal processing, whereas the spatial version of the Stroop task, which relies on right-lateralized spatial processing, has been used less frequently (e.g., Lu \& Proctor, 1995). Here, we asked participants to perform both a verbal and a spatial fouralternative forced-choice Stroop task to specifically investigate whether left lateralization of their prefrontal intrinsic brain dynamics may reflect differences in their interference resistance ability regardless of the (verbal vs. spatial) cognitive domain.

\section{METHODS}

\section{Participants}

The participants were the same as in our previous work (Ambrosini \& Vallesi, 2016). Fifty-six university students participated in the experiment after providing informed consent. All had normal or corrected-to-normal visual acuity and color vision and were reimbursed $€ 20$ for their time. The study was approved by the local bioethical committee and was conducted according to the guidelines of the Declaration of Helsinki (World Medical Association, 2013).

\section{Color-Word and Spatial Stroop Tasks}

We used a shortened version of the color-word and the spatial Stroop tasks used by Puccioni and Vallesi (2012a, 2012b, respectively; see the original studies for a detailed description of the paradigms). Briefly, the stimuli were respectively the words for four Italian color names (BLU-blue, ROSSO-red, VERDE-green, and GIALLOyellow) that were colored in one of the same four colors they denoted and arrows pointing toward one of the four corners of the screen (i.e., the upper-left, upper-right, lower-left, or lower-right corners) that were located in one of the four quadrants of the screen. The color-word and direction-location association of each stimulus was either congruent or incongruent (e.g., respectively, ROSSO presented in blue or red ink), with equal probability. The order of presentation of the stimuli was pseudorandomized so to have no repetitions of either color or meaning of the word (or direction and location of the arrow) on subsequent trials. This manipulation minimized priming confounds (see Puccioni \& Vallesi, 2012b, for details).

On each trial, the word was centrally shown for 500 msec. After a 2000-msec blank response screen, the intertrial interval varied randomly between 250 and $700 \mathrm{msec}$. Participants had to indicate the word color or arrow direction, while ignoring the word meaning or arrow location, by pressing one of four keys on the computer keyboard with their index and middle fingers of both hands. For the colorword Stroop task, the four keys were "C," "V," "B," and "N," and two color-response mappings were used, counterbalanced across participants. For the spatial Stroop task, a single mapping with four spatially arranged keys was used: "V" for lower-left, "R" for upper-left, "O" for upper-right, and "M" for lower-right.

The two Stroop tasks were performed in different sessions in the same day in randomized order. ${ }^{1}$ For each task, two blocks of 64 trials were administered; moreover, participants performed a 16-trial training block until they reached the criterion to perform the experimental task (10 correct trials).

\section{Behavioral Data Analysis}

RTs shorter than $100 \mathrm{msec}(0.06 \%)$ and from incorrect responses (7.33\%) were discarded. We log-transformed 
RTs to improve normality; moreover, to obtain robust measures of central tendency, we computed M-estimators of location (Verboven \& Hubert, 2005, 2010) for incongruent and congruent conditions. After these transformations, the variables measuring verbal and spatial Stroop effects showed acceptable skewness and kurtosis (all <.49). The Stroop effects were computed as the difference between incongruent and congruent trials; its statistical significance was assessed by means of one-sample $t$ tests against zero. The Cohen's $d$ was used as the measure of the effect size (Cohen, 1977).

\section{Resting-state EEG Recording and Analysis}

The resting-state EEG (rsEEG) and behavioral sessions were performed on different days (mean absolute interval $=23$ days, range $=1-78$ days). A single 5-min eye-closed rsEEG session was recorded at $500 \mathrm{~Hz}$ by using a 64-channel system (BrainProducts, Munich, Germany; online bandpass filter $=0.1-100 \mathrm{~Hz}$; impedances $<10 \mathrm{k} \Omega$ ) as detailed in Ambrosini and Vallesi (2016). An electrode located at FCz served as the online reference, and an electrode located at AFz served as the ground. An additional electrode placed under the left eye recorded the EOG activity.

\section{rsEEG Analysis}

Offline rsEEG processing and analyses, the source analysis, and the spectral power analysis of the estimated cortical sources activity were performed as detailed in Ambrosini and Vallesi (2016), unless otherwise specified.

Briefly, we performed the offline EEG preprocessing by using custom MATLAB (The MathWorks, Inc., Natick, MA) scripts based on functions from the EEGLAB environment (version 12.0.2b; Delorme \& Makeig, 2004). The continuous EEG data were band-pass filtered (.5-45 Hz) and submitted to an automatic channel rejection procedure based on channels probability, spectral profile, and kurtosis, which was then confirmed by visual inspection. Channels TP9 and TP10 were excluded from this and subsequent analyses because of excessive noise, and the other contaminated channels were interpolated using spherical splines. EEG data were then segmented into nonoverlapping epochs (2048 $\mathrm{msec}$ ) and re-referenced to the common average reference. Artifactual and/or outlier epochs were then rejected using an automatic procedure based on extreme values, linear trend, improbability, kurtosis, and spectral profile tests (Delorme, Sejnowski, \& Makeig, 2007; for details, see Ambrosini \& Vallesi, 2016).

Next, we performed the distributed source imaging of the preprocessed, artifact-free rsEEG epochs. We used a 15,002-dipole distributed source model derived from the standard 1-mm resolution brain of the Montreal Neurological Institute (Colin27), a forward model generated with OpenMEEG that uses a three-layer symmetric boundary element method (Gramfort, Papadopoulo, Olivi, \& Clerc, 2010; Kybic et al., 2005), and a depth-weighted minimum-norm estimation algorithm (Baillet, Mosher, \& Leahy, 2001) from the Brainstorm package (Tadel, Baillet, Mosher, Pantazis, \& Leahy, 2011). A parcellation of the cortical surface into 150 anatomical ROIs was derived in Brainstorm from the cortical atlas by Destrieux, Fischl, Dale, and Halgren (2010), which was modified to reduce the variability in number of vertices across the 148 original ROIs while ensuring a homogeneous covering of the cortical surface (Ambrosini \& Vallesi, 2016).

We then performed the spectral power analysis of the cortical source activity. In this case, we employed a different analytical strategy as compared with that used in our previous study. In particular, we first computed the power spectral densities (PSDs) of the electrical activity of each of the 15,002 vertices composing the cortical surface. This was done by using the EEGLAB (Delorme \& Makeig, 2004) spectopo function (Welch's averaged, modified periodogram method, 256-point Hamming window, 2048-point discrete Fourier transform) to obtain PSDs in the 1- to $45-\mathrm{Hz}$ frequency range with a $0.25-\mathrm{Hz}$ resolution. Then, we averaged the PSDs over the vertices composing each ROI. This improved procedure, albeit computationally very demanding (as it requires to compute, for each participant, 15,002 PSDs), significantly attenuates possible estimation errors and phase lag effects in computing the ROI PSDs, thus improving the robustness of the results, as also suggested by an anonymous reviewer. In fact, in the spectral power analysis employed in our previous article, we first estimated the mean source electrical activity for each ROI by averaging the current strength time series of each elementary dipole source within each ROI and then computed the spectral power of the mean electrical activity for each ROI. This analytical strategy was chosen to reduce the computational load, as it only requires computing PSDs for 150 vectors (i.e., the estimated mean electrical activities for each ROI) for each participant instead of the 15,002 needed in the analytical strategy employed in this study. However, the robustness of the results obtained with this strategy may potentially be biased by estimation errors in computing the PSDs of the mean ROI electrical activities and, especially, by effects due to phase lags between vertices comprised in the same ROI due to anatomical variability. Nonetheless, we report the results of the power-behavior analyses based on this procedure to facilitate the comparison of the present results with those of our previous study and thus verify our hypothesis (see Introduction).

Finally, to quantify hemispheric asymmetries in intrinsic brain dynamics, we computed the $\beta / \alpha$ hemispheric asymmetry scores $(\beta / \alpha$ HAS) for each pair of ROIs as the right-left difference in the log-transformed ratio between power in $\beta(12.5-24 \mathrm{~Hz})$ and $\alpha(7.5-12.5 \mathrm{~Hz})$ bands. For a detailed discussion about using the $\beta / \alpha$ ratio as a measure of intrinsic brain activity, the reader is referred to our previous study (Ambrosini \& Vallesi, 2016; see also Rosa, Kilner, Blankenburg, Josephs, \& Penny, 2010; Laufs et al., 2006; Kilner, Mattout, Henson, \& Friston, 2005; 
Ota, Toyoshima, \& Yamauchi, 1996). We also assessed the functional specificity of the $\beta / \alpha \_$HAS measure. Indeed, on the basis of our previous findings (Ambrosini \& Vallesi, 2016), we expected to find a specific relation between the degree of participants' prefrontal $\beta / \alpha \_$HAS and their domain-independent interference resistance ability, which is mediated by phasic cognitive control processes. To this aim, we also performed the same set of analyses on two other rsEEG asymmetry scores for which we did not find significant correlations with phasic cognitive control processes, that is, the $\beta / \theta \_H A S$ and $\gamma / \alpha \_H A S$. These measures were computed as done for the $\beta / \alpha \_$HAS (see rsEEG Analysis section) as respectively the right-left difference in the ratio between power in beta and theta $(\theta, 4-7.5 \mathrm{~Hz})$ bands and that between power in gamma $(\gamma, 24-45 \mathrm{~Hz})$ and alpha frequency bands. To the same aim, we also explored the relation between participants' interference resistance ability and the right-left hemispheric asymmetry in the $\alpha$ and $\beta$ relative power ( $\alpha_{-}$HAS and $\beta_{-}$HAS, respectively).

\section{Inferential Statistics}

We first performed a series of robust correlation analyses between either the verbal or the spatial $z$-transformed Stroop effects, respectively, and the $\beta / \alpha$ _HAS values for each ROI (both those computed based on the PSD of the mean current strength of that ROI-the same used in our previous study - and those computed based on the mean PSD over the vertices composing that ROI; see Restingstate EEG Recording and Analysis section). Specifically, we computed skipped correlations (Wilcox, 2004), which provide a robust generalization of Pearson's correlation coefficient $r$. We chose to not perform the regular Pearson's correlation analysis because, differently from the skipped correlation, (1) it does not guarantee an accurate control of the false positive rate and (2) it estimates the true correlation in the data inadequately and with a substantial loss of power when data contain outliers (especially the bivariate ones). Null hypothesis statistical significance testing for the skipped correlations was conducted using the bootstrap test (2000 resamples; two-sided 95\% confidence intervals, $\mathrm{B}_{-} \mathrm{CI}_{95 \%}$ ), which is more robust against heteroscedasticity compared with the traditional $t$ test (Pernet, Wilcox, \& Rousselet, 2012).

Moreover, because our aim was to test whether the hemispheric asymmetry in intrinsic brain dynamics (in particular, in pFC ROIs we identified in our previous study, see Ambrosini \& Vallesi, 2016) was related to Stroop performance regardless of the cognitive domain, we performed an intersection analysis to identify the ROIs for which the skipped Pearson correlation between the $\beta / \alpha_{-}$HAS and both the spatial and the verbal Stroop effect were significant in the bootstrap test, as detailed above.
Figure 1. Participants' behavioral performance in the Stroop tasks. (A, B) Scatterplots showing the participants' mean M-estimates of natural log-transformed RTs in congruent ( $x$ axis) and incongruent ( $y$ axis) trials for the verbal (A) and spatial (B) Stroop tasks. Circles represent data from each participant. The diagonal dashed line indicates the identity line $(y=x)$, so that circles above the diagonal represent participants exhibiting a Stroop effect. The boxplots in the insets show the distribution of the participants' Stroop effects; the central line/point of the box represents the median, the edges of the box are the first and third quartiles, and the whiskers represent the range of the data. (C) Scatterplot showing the participants' verbal ( $x$ axis) and spatial ( $y$ axis) Stroop effects; the dark yellow line represents the linear fit; the brown line represents the linear fit after the exclusion of one bivariate outlier (brown point). (D, E) Scatterplots showing the correlation between the participants' mean Stroop effect in the spatial (D) and verbal (E) domains ( $x$ axis) and their mixing and switching costs ( $y$ axis; cyan and purple points and lines, respectively). Note that none of the relations shown in scatterplots C-E are significant (see Behavioral Results section for details).
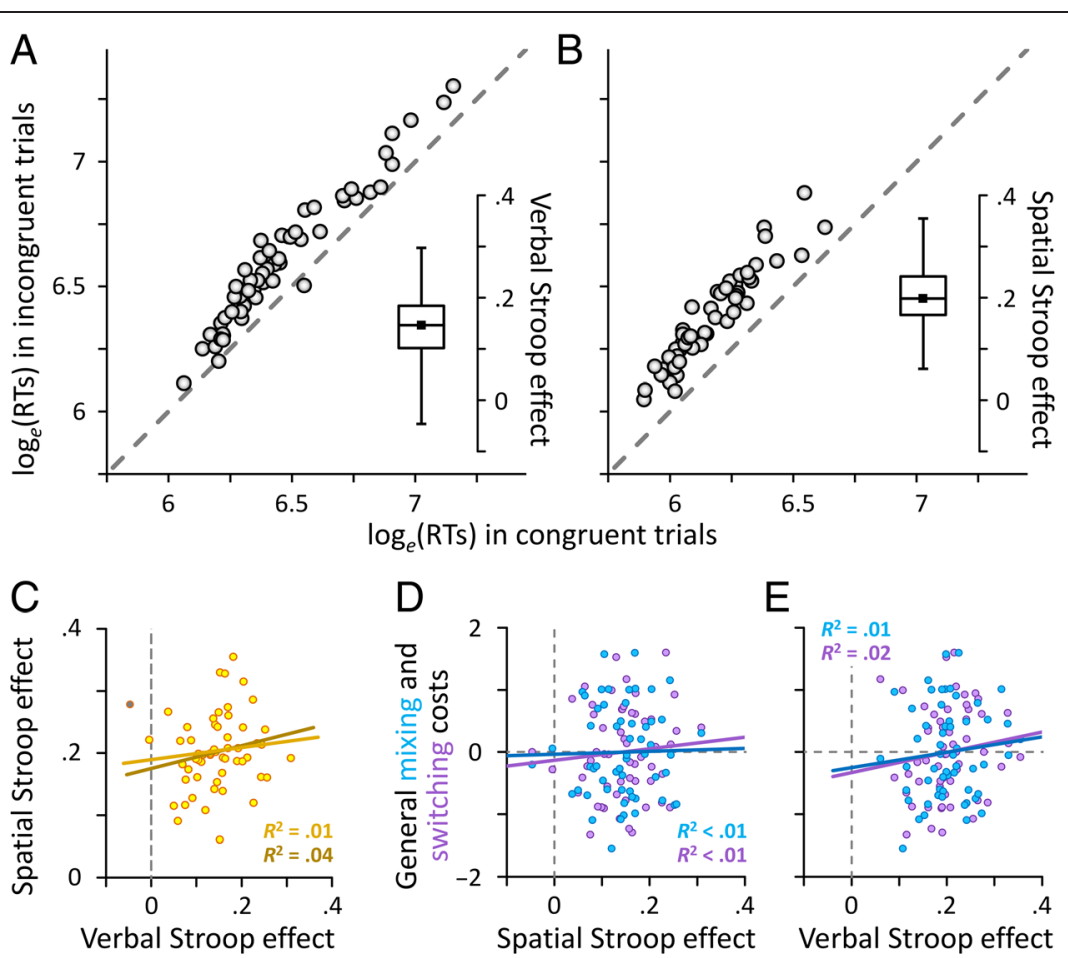

$\mathrm{E}$

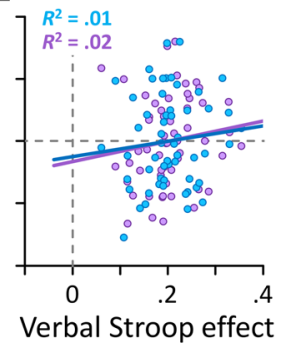


To assess the domain generality of interference resistance processes, we also computed the skipped correlation between verbal and spatial Stroop effects. Moreover, to evaluate the similarity between the participants' taskswitching and interference resistance abilities, we also computed the skipped correlations between, on the one side, the spatial and verbal Stroop effects and, on the other side, the general mixing and switching costs used in our previous study (Ambrosini \& Vallesi, 2016).

\section{RESULTS}

\section{Behavioral Results}

The analysis revealed a characteristic pattern of results, with higher RTs for the incongruent condition for both tasks, resulting in significant verbal and spatial Stroop effects (respectively, $M=0.144$ and 0.204, $S D=0.067$ and 0.060 ; both $t \mathrm{~s}(55) \geq 16$, ps $<.0001, d>2.13$; see Figure $1 \mathrm{~A}$ and $\mathrm{B}$, insets) that was shown by almost all of the participants with substantial interindividual variability (see Figure 1A and B). The spatial Stroop effect was significantly higher than the verbal one $(t(55)=$ $5.248, p>.0001, d=0.701)$, and they did not share significant variance across participants (skipped $r=$ $.192, \mathrm{~B}^{-\mathrm{CI}_{95 \%}}[-0.030,0.404]$; see Figure 1C), thus suggesting the existence of some degree of domain specificity in interference resistance processes.

It is interesting to note that neither the verbal nor the spatial Stroop effects were significantly correlated to either the general mixing and switching costs (spatial Stroop vs. mixing: skipped $r=.021, \mathrm{~B}^{-\mathrm{CI}_{95}}[-0.203,0.226]$; spatial Stroop vs. switching: skipped $r=.086, \mathrm{~B}^{-\mathrm{CI}_{95}}[-0.151$, 0.295]; verbal Stroop vs. mixing: skipped $r=.100$, B-CI $95 \%[-0.145,0.323]$; verbal Stroop vs. switching: skipped $r=.135, \mathrm{~B}^{-\mathrm{CI}_{95} \%}[-0.108,0.398]$; see Figure $1 \mathrm{D}$ and $\mathrm{E}$, respectively). These results indicate the existence of some degree of independence between the task-switching and interference resistance processes supposed to be mediated by the criterion-setting executive function.

\section{Power-Behavior Correlations}

\section{Spectral Power of the Mean Electrical Activity for Each ROI}

To facilitate the comparison of the present results with those of our previous study, we first carried out a robust correlation analysis based on the ROIs spectral power computed using the same procedure as in our previous study (see rsEEG Analysis section). A number of ROIs showed significant correlation between $\beta / \alpha \_$HAS and either the verbal or spatial interference resistance, suggesting that the participants' hemispheric asymmetry in intrinsic rsEEG spectral activity can reflect their differences in interference resistance abilities with some degree of domain specificity. However, our specific purpose was to identify the cortical regions showing hemispheric asymmetry in intrinsic brain
Table 1. Results of the Confirmatory Intersection Analysis

\begin{tabular}{|c|c|c|c|c|c|c|c|c|}
\hline \multirow[b]{2}{*}{$R O I$} & \multicolumn{4}{|c|}{ Verbal Stroop Task } & \multicolumn{4}{|c|}{ Spatial Stroop Task } \\
\hline & \#Out & $r_{s k i}$ & ${ }_{L B} C I_{95 \%}$ & ${ }_{U B} C I_{95 \%}$ & \#Out & $r_{s k i}$ & ${ }_{L B} C I_{95 \%}$ & ${ }_{U B} C I_{95 \%}$ \\
\hline mpSFG & 5 & .246 & 0.002 & $0.494 *$ & 2 & .334 & 0.053 & $0.550 *$ \\
\hline mMFG & 3 & .326 & 0.050 & $0.578 *$ & 1 & .328 & 0.098 & $0.536 *$ \\
\hline
\end{tabular}

\#Out $=$ number of data points identified as outliers; $r_{\mathrm{ski}}=$ skipped Pearson's correlation; ${ }_{\mathrm{LB}} \mathrm{CI}_{95 \%}$ and ${ }_{\mathrm{UB}} \mathrm{CI}_{95 \%}=$ lower and upper bound of the $95 \%$ bootstrap confidence interval, respectively; $\mathrm{mpSFG}=$ mid-posterior part of the superior frontal gyrus; $\mathrm{mMFG}=$ middle part of the middle frontal gyrus.

$* p<.05$ at the nonparametric percentile bootstrap test (see Inferential Statistics section).

dynamics that was significantly related to Stroop performance in both cognitive domains. The results of the subsequent confirmatory intersection analysis showed that this was true for two ROIs in pFC (see Table 1), namely the mid-posterior superior frontal gyrus (mpSFG), which included the pre-SMA, and the middle part of the middle frontal gyrus (mMFG) ${ }^{2}$ For both ROIs, participants who showed higher $\beta / \alpha$ power in the left than right hemisphere also had significantly smaller Stroop effects in both verbal and spatial tasks. In other words, participants with a strongly left-lateralized brain activity at rest (i.e., with more negative $\beta / \alpha \_$HAS values) in either the $\mathrm{mpSFG} /$ pre-SMA or the mMFG were more able to exert cognitive control to resist interference from irrelevant information and prepotent responses, regardless of the cognitive domain.

\section{Mean Spectral Power over Vertices Composing Each ROI}

Next, to improve the robustness of the results and confirm those revealed by the preceding analysis, we performed a robust correlation analysis based on the improved procedure to compute the ROI-based spectral power (see rsEEG Analysis section). Again, a number of ROIs showed significant correlation between $\beta / \alpha \_$HAS and either the verbal or spatial interference resistance (see Table 2 and Figure 2). However, these domain-specific effects fall beyond the scope of this study and are not discussed further.

The intersection analysis identified five cortical regions showing hemispheric asymmetry in intrinsic brain dynamics that was significantly related to both verbal and spatial Stroop performance (see Table 2 and Figure 3). Two of these were the same prefrontal ROIs as those emerged in the intersection analysis based on the ROIs' spectral power computed using the same procedure as in our previous study, namely the mpSFG and the mMFG. The intersection analysis also revealed two other prefrontal ROIs that were located caudally to the mMFG, that is, the posterior portion of the middle frontal gyrus and the inferior portion of the precentral sulcus, which includes the socalled inferior frontal junction (IFJ). The fifth ROI was the paracentral lobule. For all these ROIs, participants with a strongly left-lateralized brain activity at rest (i.e., showing higher $\beta / \alpha$ power in the left than right hemisphere) also 
Table 2. Results of the Power-Behavior Robust Correlation Analysis Based on the Improved Procedure

\begin{tabular}{|c|c|c|c|c|c|c|c|c|}
\hline \multirow[b]{2}{*}{$R O I$} & \multicolumn{4}{|c|}{ Verbal Stroop Task } & \multicolumn{4}{|c|}{ Spatial Stroop Task } \\
\hline & \#Out & $r_{s k i}$ & ${ }_{L B} C I_{95 \%}$ & ${ }_{U B} C I_{95 \%}$ & \#Out & $r_{s k i}$ & ${ }_{L B} C I_{95 \%}$ & ${ }_{U B} C I_{95 \%}$ \\
\hline $\mathrm{mpSFG}^{\mathrm{a}}$ & 1 & .368 & 0.204 & $0.536^{*}$ & 2 & .235 & 0.030 & $0.444^{*}$ \\
\hline $\mathrm{mMFG}^{\mathrm{a}}$ & 3 & .289 & 0.026 & $0.501 *$ & 3 & .424 & 0.169 & $0.621 *$ \\
\hline $\mathrm{pMFG}^{\mathrm{a}}$ & 4 & .279 & 0.051 & $0.506^{*}$ & 2 & .246 & 0.037 & $0.453 *$ \\
\hline infPreCS $^{\mathrm{a}}$ & 3 & .302 & 0.061 & $0.506^{*}$ & 1 & .249 & 0.025 & $0.455^{*}$ \\
\hline $\mathrm{PCL}^{\mathrm{a}}$ & 1 & .211 & 0.059 & $0.362^{*}$ & 3 & .436 & 0.217 & $0.611 *$ \\
\hline CircInS & 5 & .387 & 0.123 & $0.608^{*}$ & 2 & -.082 & -0.330 & 0.160 \\
\hline MCingS & 1 & .299 & 0.041 & $0.520 *$ & 1 & -.113 & -0.335 & 0.114 \\
\hline lOrS & 3 & .239 & 0.005 & $0.429^{*}$ & 1 & .100 & -0.150 & 0.366 \\
\hline Tpole & 4 & -.275 & -0.501 & $-0.012^{*}$ & 0 & .109 & -0.120 & 0.337 \\
\hline aMCC & 1 & .192 & -0.049 & 0.415 & 3 & .268 & 0.105 & $0.434 *$ \\
\hline PreCun & 1 & -.047 & -0.259 & 0.174 & 5 & .398 & 0.211 & $0.545 *$ \\
\hline TransFP & 1 & .069 & -0.212 & 0.335 & 5 & -.257 & -0.468 & $-0.034^{*}$ \\
\hline
\end{tabular}

\#Out $=$ number of data points identified as outliers; $r_{\text {ski }}=$ skipped Pearson's correlation; ${ }_{\mathrm{LB}} \mathrm{CI}_{95 \%}$ and ${ }_{\mathrm{UB}} \mathrm{CI}_{95 \%}=10 \mathrm{wer}$ and $\mathrm{upper}$ bound of the $95 \%$ bootstrap confidence interval, respectively; mpSFG = mid-posterior part of the superior frontal gyrus; mMFG $=$ middle part of the middle frontal gyrus; $\mathrm{pMFG}=$ posterior part of the middle frontal gyrus; infPreCS $=$ inferior part of the precentral sulcus; PCL $=$ paracentral lobule; CircInS $=$ circular insular sulcus; MCingS = marginal cingulate sulcus; lOrS = lateral orbital sulcus; Tpole = temporal pole; aMCC = anterior part of the middle cingulate cortex; PreCun $=$ precuneus; TransFP $=$ transverse frontopolar sulcus and gyrus .

${ }^{a}$ Significant at the intersection analysis.

$* p<.05$ at the nonparametric percentile bootstrap test (see Inferential Statistics section).

had significantly smaller Stroop effects in both verbal and spatial tasks (Figure 3), that is, they were more able to exert cognitive control to resist interference from irrelevant information and prepotent responses, regardless of the cognitive domain.

\section{Other Spectral Measures}

The intersection analysis on the $\gamma / \alpha \_$HAS did not identify any ROI showing significant correlation between this spectral measure and the participants' interference resistance ability in both verbal and spatial domains. Conversely, the participants' performance in both verbal and spatial Stroop tasks was significantly related to their $\beta / \theta$ HAS in the posterior part of the middle cingulate cortex. In particular, participants who showed a stronger left lateralization in the $\beta / \theta$ power ratio (i.e., those showing more negative $\beta / \theta$ HAS values) in this cortical region also had smaller Stroop effects in both the verbal and spatial domains (respectively, skipped correlation $=.242$ and
Figure 2. Results of the power-behavior robust correlation analysis. The figure shows the cortical surface of the left hemisphere showing the ROIs detected by the power-behavior robust correlation analysis for both the verbal (in green) and spatial (in red) Stroop effects, respectively. In dark yellow are shown the ROIs detected by both the intersection analyses based on our previous procedure and on the improved procedure to compute ROIs PSDs, namely the mid-posterior part of the superior frontal gyrus (mpSFG) and the middle part of the middle frontal gyrus (mMFG). In yellow are shown the ROIs detected by the intersection analysis based on the improved procedure, namely the posterior part of the middle frontal gyrus (pMFG), the inferior part of the precentral sulcus (infPreCS), and the paracentral lobule (PCL). See Table 2 for other abbreviations. 
Figure 3. Results of the intersection analysis. The figure shows the cortical surface of the left hemisphere showing the ROIs detected by the intersection analysis.

The scatterplots show the corresponding correlations between the participants' hemispheric asymmetry of rsEEG spectral activity $\left(\beta / \alpha \_\right.$HAS, $x$ axis) and their z-transformed Stroop effects ( $y$ axis) in both the verbal and spatial Stroop tasks (light green and red points,

respectively). Positive values of $\beta / \alpha \_H A S$ indicate a stronger right-lateralized brain activity at rest (see rsEEG Analysis section). The green and red regression lines reflect the skipped Pearson correlation for the verbal and spatial Stroop

effects, respectively. Darker diamonds indicate the outliers excluded by the robust correlation analyses. PCL $=$ paracentral lobule; $\mathrm{mpSFG}=$ mid-posterior part of the superior frontal gyrus; $\mathrm{mMFG}=$ middle part of the middle frontal gyrus; $\mathrm{pMFG}=$ posterior part of the middle frontal gyrus; InfPreCS $=$ inferior precentral sulcus.

243, two-sided 95\% confidence intervals, $\mathrm{B}_{-} \mathrm{CI}_{95 \%}[0.027$, $0.472]$ and $[0.008,0.472])$, that is, they were more able to exert phasic cognitive control processes to resist to interfering information.

As regards the intersection analyses on the $\alpha$ and $\beta$ relative power asymmetries, no ROIs showed significant domain-independent power-behavior correlations for the $\alpha$ _HAS measure. Conversely, the participants' interference resistance ability in both verbal and spatial domains was significantly related to their $\beta$ _HAS in the mMFG, that is, one of the ROIs for which we found a significant domain-independent power-behavior correlation for the $\beta / \alpha$ HAS measure. In particular, participants who showed more negative (i.e., more left-lateralized) $\beta$ _HAS values in this prefrontal region also had smaller Stroop effects in both the verbal and spatial domains (respectively, skipped correlation $=.252$ and $.445, \mathrm{~B}_{-} \mathrm{CI}_{95 \%}[0.030,0.464]$ and $[0.178,0.647])$, that is, they were more able to exert phasic cognitive control processes to resist to interfering information.

\section{DISCUSSION}

In this study, we employed an interindividual difference approach to test whether participants' hemispheric asymmetry in resting-state electrophysiological brain dynamics may represent one of the underlying causes of the huge individual differences in executive control performance and, specifically, in the interference resistance ability. In doing this, we aimed to test the hypothesis of a domainindependent, left-lateralized $\mathrm{pFC}$ specialization for phasic cognitive control processes mediating interference resis- tance abilities and, thus, Stroop performance (Vallesi, 2012), as implied by the ROBBIA model of executive functions (Stuss, 2011; Stuss \& Alexander, 2007). To this aim, we recorded participants' rsEEG activity and performed a spectral power analysis on the estimated cortical source activity. We measured participants' lateralized brain dynamics at rest as the right-left hemispheric asymmetry score for the $\beta / \alpha$ power ratio by using the same procedure as in our previous study (Ambrosini \& Vallesi, 2016), as well as a procedure that improves the robustness of the results. To measure participants' domain-general interference resistance ability, verbal and spatial Stroop tasks were used. Robust correlations followed by intersection analyses showed that participants who were more able to inhibit irrelevant information in both domains also exhibited a stronger resting-staterelated left-lateralized activity in a cluster of pFC regions, namely the mpSFG/pre-SMA, the middle and posterior parts of the middle frontal gyrus (MFG), and the inferior precentral sulcus/IFJ.

In line with our previous findings (Ambrosini \& Vallesi, 2016), the present results thus suggest that a more strongly left-lateralized intrinsic prefrontal activity may represent a stable individual difference factor leading to biases in engaging the left-lateralized cognitive control processes to boost the processing of task-relevant information while suppressing that of irrelevant and interfering one. Specifically, the smaller Stroop effects observed in individuals with stronger left-lateralized $\mathrm{pFC} \beta / \alpha$ at rest would be, at least in part, the consequence of displaying a specific brain state at rest characterized by greater activity in the left prefrontal nodes of the frontoparietal circuit 
supposedly involved in criterion-setting processes (Vallesi, 2012; Stuss \& Alexander, 2007), which would in turn facilitate the activation of the same cortical circuit during the execution of Stroop tasks, leading to a better performance in exerting interference resistance processes. In other words, the asymmetry in the resting-state-related EEG spectral profile in a pFC region would play a possible role as a prior for both task-related activity and the behavioral performance in tasks tapping into the cognitive processes mediated by that region (cf. Spadone et al., 2015; Raichle, 2011).

pFC areas we found to show a significant powerbehavior correlation for both the verbal and the spatial Stroop tasks are consistent with previous fMRI literature. Indeed, differential activation patterns induced by interference resistance processes are most commonly reported in the prefrontal and inferior parietal region, with a clear left lateralization, and in the anterior cingulate and superior medial pFC bilaterally (cf. Zysset, Müller, Lohmann, \& von Cramon, 2001), as shown by a number of meta-analyses of fMRI studies on Stroop performance (Neumann, von Cramon, \& Lohmann, 2008; Laird, Fox, et al., 2005; Laird, McMillan, et al., 2005; Neumann, Lohmann, Derrfuss, \& von Cramon, 2005). In particular, the left MFG, pre-SMA, and IFJ corresponding to pFC ROIs that we found here were three of the five activation foci composing what Neumann et al. (2005) called the "dominant network" of cortical areas that jointly show significant activation across fMRI Stroop studies. Note that the remaining two foci of this network were located in the left and right ACC, thus showing no hemispheric specialization; this could be the reason why our analyses failed to detect a correlation between Stroop performance and the asymmetry in the intrinsic spectral activity of the ACC. Interestingly, a greater task-related activity in the left MFG has also been associated with better interference resistance ability specifically in an unblocked, uncued context that maximally taxes criterion-setting processes (Floden, Vallesi, \& Stuss, 2011).

The critical role of left $\mathrm{pFC}$ in interference resistance processes has also been shown in neuropsychological studies on patients with pFC focal damage. Indeed, patients with left pFC lesions have impaired Stroop performance (Tsuchida \& Fellows, 2013; Demakis, 2004; Stuss, Floden, Alexander, Levine, \& Katz, 2001; Perret, 1974). Our results thus extend previous findings in providing support for the idea of a left $\mathrm{pFC}$ hemispheric asymmetry of cognitive control processes mediating participants' interference resistance abilities regardless of the cognitive domain, that is, not only when performing the classic color-word Stroop task, which strongly relies on left-lateralized verbal processing, but also when performing a version of the Stroop task based on visuospatial information, which relies on right-lateralized spatial processing.

It is important to note that one of pFC ROIs we found to show a significant power-behavior correlation for the domain-general Stroop performance, namely the mMFG, is the same we found to show a significant power-behavior correlation for the domain-general task-switching performance in the same participants (Ambrosini \& Vallesi, 2016). This was confirmed both by the analysis employing the same analytical procedure as that used in our previous work and by the one using a slightly different procedure to improve the robustness of the results. In other words, the interindividual variability in our participants' performance when performing both the Stroop and task-switching tasks was related to the same neurophysiological individual difference factor, that is, the hemispheric asymmetry in the intrinsic activity of that pFC ROI, as assessed by its EEG spectral profile at rest. Moreover, the left lateralization of pFC activity at rest seems to be an individual difference factor specifically related to the ability in exerting phasic cognitive control processes, whereas the ability in exerting sustained cognitive control processes would be related to a right lateralization of pFC activity at rest, as shown in our previous study (Ambrosini \& Vallesi, 2016).

Taken together, the present results confirm our hypothesis and provide support for the proposed model of a left-right prefrontal fractionation of distinct executive functions (Vallesi, 2012; Stuss \& Alexander, 2007). This model, indeed, proposes a left $\mathrm{pFC}$ specialization for the criterion setting (or task setting), that is, the executive function that is supposed to mediate both the task-switching and the interference resistance ability. Indeed, criterion setting has been defined as the phasic, transient cognitive control processes needed to set up or select task-relevant rules (Stuss \& Alexander, 2007) and suppress the task-irrelevant rules and information that may interfere with accomplishing the goal-driven task at hand (Vallesi et al., 2012; Alexander, Stuss, Picton, Shallice, \& Gillingham, 2007; also see Thompson-Schill et al., 1997).

Therefore, our results suggest that the relation we found between the interindividual variability in the leftlateralized $\mathrm{pFC}$ intrinsic activity and the interindividual variability in exerting phasic cognitive control can be generalized - at least in part-not only to the different cognitive domains (i.e., verbal vs. spatial) but also to different criterion-setting subprocesses in a specific way. This is also supported both by meta-analyses on fMRI studies using different tasks, including the Stroop and task-switching ones, to assess different executive subprocesses (Nee, Wager, \& Jonides, 2007; Derrfuss, Brass, Neumann, \& von Cramon, 2005) and by an fMRI study directly investigating Stroop and task-switching performance in a within-session within-group design (Derrfuss, Brass, \& von Cramon, 2004). The authors found common activations with a clear left lateralization in a network of prefrontal, parietal, and subcortical regions, including three foci in the left MFG, pre-SMA/medial superior frontal gyrus, and IFJ that overlap with pFC ROIs we found in this study (Derrfuss et al., 2004).

However, the fact that we found a similar pattern of significant mMFG-related $\beta / \alpha$ _HAS-behavior correlations both across verbal and spatial domains (in interference 
resistance ability) and across task-switching and Stroop tasks (in phasic cognitive control processes) seems not to be simply due to shared interindividual variability in behavioral performance. If the behavioral measures were all strongly correlated between each other, indeed, it would have been not surprising at all to find similar mMFG-related $\beta / \alpha$ _HAS-behavior correlations across domains and/or tasks. Nevertheless, the behavioral results showed that our measures are not significantly correlated. ${ }^{3}$ To note, the left lateralization of pFC activity at rest would be only one of the many possible individual difference factors that co-contribute in mediating our phasic cognitive control ability; moreover, it could also act as a moderator variable in the complex pattern of relations between individual difference factors and behavioral measures of executive functioning, in line with our proposed explanation of the functional relevance of our results (see above and Ambrosini \& Vallesi, 2016). Additional research is nonetheless necessary to clarify the complex pattern of factors contributing to explain individual differences in executive functioning.

It should also be noted that, differently from our previous results concerning task-switching ability, we here found that the domain-independent interference resistance ability is related to the resting-state $\beta / \alpha$ hemispheric asymmetries in a larger cluster of $\mathrm{pFC}$ regions, thus suggesting the existence of some degree of task specificity in the interplay between neurophysiological individual difference factors and domain-general phasic cognitive control processes. In particular, the present results suggest the involvement of the medial superior frontal gyrus and, especially, the IFJ in phasic cognitive control, in line with previous findings (Derrfuss et al., 2004, 2005), suggesting that the intrinsic activity in different $\mathrm{pFC}$ regions may be related to distinct subprocesses in mediating phasic cognitive control ability.

The present results also add to previous findings in highlighting the importance of the prefrontal lateralization of spectral dynamics in the beta band at rest. Indeed, we also found that the participants' interference resistance ability in both verbal and spatial domains was significantly related to their $\beta$ _HAS in the mMFG, that is, the same ROI for which we found a significant correlation between the $\beta / \alpha \_$HAS measure and both the domainindependent Stroop performance, in this study, and the domain-independent task-switching performance, in our previous study (Ambrosini \& Vallesi, 2016). This is consistent with the idea that a shift in the EEG spectral profile toward beta frequencies in a given cortical region (i.e., an enhancement in both its $\beta / \alpha$ and $\beta / \theta$ power ratio) would be related to its BOLD activation (Rosa et al., 2010; Kilner et al., 2005).

To conclude, this study confirms and extends our previous findings by showing that interindividual differences in prefrontal hemispheric asymmetry in EEG spectral profile at rest, that is, the same neurophysiological individual difference factor we showed to be related to domain-general task-switching performance (Ambrosini \& Vallesi, 2016), also explains interindividual variability in interference resistance ability. The present data thus provide support for the hypothesis of a left prefrontal functional specialization for the phasic cognitive control abilities mediated by the criterion-setting processes.

\section{Acknowledgments}

The authors are funded by the European Research Council Starting Grant LEX-MEA 313692 (FP7/2007-2013) to A. V. The authors thank Città della Speranza, Padova, for its logistic support and Sandra Arbula and Mariagrazia Capizzi for their assistance in data collection.

Reprint requests should be sent to Ettore Ambrosini, Department of Neuroscience, University of Padua, Via Giustiniani, 5, 35128 Padova, Italy, or via e-mail: ettore.ambrosini@unipd.it.

\section{Notes}

1. In the same session, participants performed additional behavioral tasks tapping into different executive functions, which were not the object of this study and whose results will be reported elsewhere.

2. The mMFG ROI also showed significant nonrobust (i.e., standard) Pearson's correlations between participants' $\beta / \alpha$ HAS and Stroop performance in both cognitive domains, thus showing that this result was not influenced by the removal of outliers.

3. Note that it is plausible to have a latent variable (in our case, the construct of phasic cognitive control ability) that is significantly related to a number of manifest variables (in our case, the different behavioral measures) that, in turn, are not significantly correlated between each other. This is especially true when the latent-manifest correlations are modest in magnitude, as in our case.

\section{REFERENCES}

Alexander, M. P., Stuss, D. T., Picton, T., Shallice, T., \& Gillingham, S. (2007). Regional frontal injuries cause distinct impairments in cognitive control. Neurology, 68, 1515-1523.

Ambrosini, E., \& Vallesi, A. (2016). Asymmetry in prefrontal resting-state EEG spectral power underlies individual differences in phasic and sustained cognitive control. Neuroimage, 124, 843-857.

Baillet, S., Mosher, J. C., \& Leahy, R. M. (2001). Electromagnetic brain mapping. IEEE Signal Processing Magazine, 18, 14-30.

Braver, T. S. (2012). The variable nature of cognitive control: A dual mechanisms framework. Trends in Cognitive Sciences, 16, 106-113.

Braver, T. S., Cole, M. W., \& Yarkoni, T. (2010). Vive les differences! Individual variation in neural mechanisms of executive control. Current Opinion in Neurobiology, 20, 242-250.

Burgess, P. W., \& Shallice, T. (1996). Response suppression, initiation and strategy use following frontal lobe lesions. Neuropsychologia, 34, 263-272.

Cabeza, R., \& Nyberg, L. (2003). Seeing the forest through the trees: The cross-function approach to imaging cognition. In A. Zani \& A. M. Proverbio (Eds.), The cognitive electrophysiology of mind and brain (pp. 41-68). San Diego: Academic Press

Cohen, J. (1977). Statistical power analysis for the behavioral sciences (rev. ed.). Hillsdale, NJ: Lawrence Erlbaum Associates, Inc. 
Delorme, A., \& Makeig, S. (2004). EEGLAB: An open source toolbox for analysis of single-trial EEG dynamics including independent component analysis. Journal of Neuroscience Methods, 134, 9-21.

Delorme, A., Sejnowski, T., \& Makeig, S. (2007). Enhanced detection of artifacts in EEG data using higher-order statistics and independent component analysis. Neuroimage, 34, $1443-1449$.

Demakis, G. J. (2004). Frontal lobe damage and tests of executive processing: A meta-analysis of the category test, Stroop test, and trail-making test. Journal of Clinical and Experimental Neuropsychology, 26, 441-450.

Derrfuss, J., Brass, M., Neumann, J., \& von Cramon, D. Y. (2005). Involvement of the inferior frontal junction in cognitive control: Meta-analyses of switching and Stroop studies. Human Brain Mapping, 25, 22-34.

Derrfuss, J., Brass, M., \& von Cramon, D. Y. (2004). Cognitive control in the posterior frontolateral cortex: Evidence from common activations in task coordination, interference control, and working memory. Neuroimage, 23, 604-612.

Destrieux, C., Fischl, B., Dale, A., \& Halgren, E. (2010). Automatic parcellation of human cortical gyri and sulci using standard anatomical nomenclature. Neuroimage, 53, 1-15.

Duncan, J., \& Owen, A. M. (2000). Common regions of the human frontal lobe recruited by diverse cognitive demands. Trends in Neurosciences, 23, 475-483.

Fletcher, P. C., Shallice, T., \& Dolan, R. J. (2000). "Sculpting the response space"-An account of left prefrontal activation at encoding. Neuroimage, 12, 404-417.

Floden, D., Vallesi, A., \& Stuss, D. T. (2011). Task context and frontal lobe activation in the Stroop task. Journal of Cognitive Neuroscience, 23, 867-879.

Gramfort, A., Papadopoulo, T., Olivi, E., \& Clerc, M. (2010). OpenMEEG: Opensource software for quasistatic bioelectromagnetics. BioMedical Engineering OnLine, 9, 45.

Hellige, J. B. (1993). Hemispheric asymmetry: What's right and what's left (Vol. 6). Cambridge, MA: Harvard University Press.

Kilner, J. M., Mattout, J., Henson, R., \& Friston, K. J. (2005). Hemodynamic correlates of EEG: A heuristic. Neuroimage, 28, 280-286.

Koechlin, E., Ody, C., \& Kouneiher, F. (2003). The architecture of cognitive control in the human prefrontal cortex. Science, 302, 1181-1185.

Kybic, J., Clerc, M., Abboud, T., Faugeras, O., Keriven, R., \& Papadopoulo, T. (2005). A common formalism for the integral formulations of the forward EEG problem. IEEE Transactions on Medical Imaging, 24, 12-28.

Laird, A. R., Fox, P. M., Price, C. J., Glahn, D. C., Uecker, A. M., Lancaster, J. L., et al. (2005). ALE meta-analysis: Controlling the false discovery rate and performing statistical contrasts. Human Brain Mapping, 25, 155-164.

Laird, A. R., McMillan, K. M., Lancaster, J. L., Kochunov, P., Turkeltaub, P. E., Pardo, J. V., et al. (2005). A comparison of label-based review and ALE meta-analysis in the Stroop task. Human Brain Mapping, 25, 6-21.

Laufs, H., Holt, J. L., Elfont, R., Krams, M., Paul, J. S., Krakow, K., et al. (2006). Where the BOLD signal goes when alpha EEG leaves. Neuroimage, 31, 1408-1418.

Lu, C. H., \& Proctor, R. W. (1995). The influence of irrelevant location information on performance: A review of the Simon and spatial Stroop effects. Psychonomic Bulletin \& Review, 2, 174-207.

MacDonald, A. W., Cohen, J. D., Stenger, V. A., \& Carter, C. S. (2000). Dissociating the role of the dorsolateral prefrontal and anterior cingulate cortex in cognitive control. Science, 288, 1835-1838.
MacLeod, C. M. (1991). Half a century of research on the Stroop effect: An integrative review. Psychological Bulletin, 109, 163.

Miller, E. K., \& Cohen, J. D. (2001). An integrative theory of prefrontal cortex function. Annual Review of Neuroscience, 24, 167-202.

Nee, D. E., Wager, T. D., \& Jonides, J. (2007). Interference resolution: Insights from a meta-analysis of neuroimaging tasks. Cognitive, Affective \& Behavioral Neuroscience, 7, $1-17$.

Neumann, J., Lohmann, G., Derrfuss, J., \& von Cramon, D. Y. (2005). Meta-analysis of functional imaging data using replicator dynamics. Human Brain Mapping, 25, 165-173.

Neumann, J., von Cramon, D. Y., \& Lohmann, G. (2008). Model-based clustering of meta-analytic functional imaging data. Human Brain Mapping, 29, 177-192.

Norman, D. A., \& Shallice, T. (1986). Attention to action. In R. J. Davidson, G. E. Schwartz, \& D. Shapiro (Eds.), Consciousness and self-regulation: Advances in research and theory (Vol. 4, pp. 1-18). Boston, MA: Springer US.

Ota, T., Toyoshima, R., \& Yamauchi, T. (1996). Measurements by biphasic changes of the alpha band amplitude as indicators of arousal level. International Journal of Psychophysiology, 24, 25-37.

Pernet, C. R., Wilcox, R., \& Rousselet, G. A. (2012). Robust correlation analyses: False positive and power validation using a new open source Matlab toolbox. Frontiers in Psychology, 3, 606.

Perret, E. (1974). The left frontal lobe of man and the suppression of habitual responses in verbal categorical behaviour. Neuropsychologia, 12, 323-330.

Puccioni, O., \& Vallesi, A. (2012a). High cognitive reserve is associated with a reduced age-related deficit in spatial conflict resolution. Frontiers in Human Neuroscience, 6, 327.

Puccioni, O., \& Vallesi, A. (2012b). Sequential congruency effects: Disentangling priming and conflict adaptation. Psychological Research, 76, 591-600.

Raichle, M. E. (2011). The restless brain. Brain Connectivity, 1, 3-12.

Rosa, M. J., Kilner, J., Blankenburg, F., Josephs, O., \& Penny, W. (2010). Estimating the transfer function from neuronal activity to BOLD using simultaneous EEG-fMRI. Neuroimage, 49, 1496-1509.

Shallice, T., Stuss, D. T., Picton, T. W., Alexander, M. P., \& Gillingham, S. (2007). Multiple effects of prefrontal lesions on task-switching. Frontiers in Human Neuroscience, 1, 2.

Shallice, T., Stuss, D. T., Picton, T. W., Alexander, M. P., \& Gillingham, S. (2008). Mapping task switching in frontal cortex through neuropsychological group studies. Frontiers in Neuroscience, 2, 79-85.

Spadone, S., Della Penna, S., Sestieri, C., Betti, V., Tosoni, A., Perrucci, M. G., et al. (2015). Dynamic reorganization of human resting-state networks during visuospatial attention. Proceedings of the National Academy of Sciences, U.S.A., 112, 8112-8117.

Stroop, J. R. (1935). Studies of interference in serial verbal reactions. Journal of Experimental Psychology, 18, 643.

Stuss, D., Shallice, T., Alexander, M., \& Picton, T. (1995). A multidisciplinary approach to anterior attentional functions. Annals of the New York Academy of Sciences, 769, 191-212.

Stuss, D. T. (2011). Functions of the frontal lobes: Relation to executive functions. Journal of the International Neuropsychological Society, 17, 759-765.

Stuss, D. T., \& Alexander, M. P. (2007). Is there a dysexecutive syndrome? Philosophical Transactions of the Royal Society of London, Series B, Biological Sciences, 362, 901-915. 
Stuss, D. T., Floden, D., Alexander, M. P., Levine, B., \& Katz, D. (2001). Stroop performance in focal lesion patients: Dissociation of processes and frontal lobe lesion location. Neuropsychologia, 39, 771-786.

Tadel, F., Baillet, S., Mosher, J. C., Pantazis, D., \& Leahy, R. M. (2011). Brainstorm: A user-friendly application for MEG/EEG analysis. Computational Intelligence and Neuroscience, 2011, 879716.

Thompson-Schill, S. L., D'Esposito, M., Aguirre, G., \& Farah, M. J. (1997). Role of left inferior prefrontal cortex in retrieval of semantic knowledge: A reevaluation. Proceedings of the National Academy of Sciences, U.S.A., 94, 14792-14797.

Tsuchida, A., \& Fellows, L. K. (2013). Are core component processes of executive function dissociable within the frontal lobes? Evidence from humans with focal prefrontal damage. Cortex, 49, 1790-1800.

Vallesi, A. (2012). Organisation of executive functions: Hemispheric asymmetries. Journal of Cognitive Psychology, 24, 367-386.
Vallesi, A., McIntosh, A. R., Crescentini, C., \& Stuss, D. T. (2012). fMRI investigation of speed-accuracy strategy switching. Human Brain Mapping, 33, 1677-1688.

Verboven, S., \& Hubert, M. (2005). LIBRA: A MATLAB library for robust analysis. Chemometrics and Intelligent Laboratory Systems, 75, 127-136.

Verboven, S., \& Hubert, M. (2010). Matlab library LIBRA. Wiley Interdisciplinary Reviews: Computational Statistics, 2, 509-515.

Wilcox, R. (2004). Inferences based on a skipped correlation coefficient. Journal of Applied Statistics, 31, 131-143.

World Medical Association. (2013). World Medical Association Declaration of Helsinki: Ethical principles for medical research involving human subjects. JAMA, 310, 2191-2194.

Zysset, S., Müller, K., Lohmann, G., \& von Cramon, D. Y. (2001). Color-word matching Stroop task: Separating interference and response conflict. Neuroimage, 13, 29-36. 